Article

\title{
Some New Families of Special Polynomials and Numbers Associated with Finite Operators
}

\author{
Yilmaz Simsek
}

Department of Mathematics, Faculty of Science, University of Akdeniz, Antalya TR-07058, Turkey; ysimsek@akdeniz.edu.tr

Received: 26 December 2019; Accepted: 29 January 2020; Published: 4 February 2020

check for updates

\begin{abstract}
The aim of this study was to define a new operator. This operator unify and modify many known operators, some of which were introduced by the author. Many properties of this operator are given. Using this operator, two new classes of special polynomials and numbers are defined. Many identities and relationships are derived, including these new numbers and polynomials, combinatorial sums, the Bernoulli numbers, the Euler numbers, the Stirling numbers, the Daehee numbers, and the Changhee numbers. By applying the derivative operator to these new polynomials, derivative formulas are found. Integral representations, including the Volkenborn integral, the fermionic $p$-adic integral, and the Riemann integral, are given for these new polynomials.
\end{abstract}

Keywords: generating function; bernoulli numbers; euler numbers; stirling numbers; central factorial numbers; daehee numbers; changhee numbers; special functions; operators, $p$-adic integral

MSC: 12D10; 11B68; 11S40; 11S80; 26C05; 26C10; 30B40; 30C15

\section{Introduction}

Special polynomials, special numbers, special functions, and operators are widely used in mathematics, physics, and engineering. Our motivation was to construct new classes special polynomials and numbers with the help of an operator. By applying a derivative operator and $p$-adic integrals to these new special polynomials, many interesting identities, relations, and formulas were found. The results of this paper include some well-known special numbers, such as the Bernoulli numbers, the Cauchy numbers, the Euler numbers, the Stirling numbers, the Daehee numbers, and the Changhee numbers.

The following notations and definitions are used throughout this paper: Let

$$
\mathbb{N}=\{1,2,3, \ldots\}, \quad \mathbb{N}_{0}=\mathbb{N} \cup\{0\} .
$$

Let $\mathbb{Z}$ denote the set of integers, $\mathbb{R}$ denote the set of real numbers, and $\mathbb{C}$ denote the set of complex numbers.

$$
0^{n}= \begin{cases}1, & (n=0) \\ 0, & (n \in \mathbb{N})\end{cases}
$$

and

$$
\left(\begin{array}{l}
\lambda \\
0
\end{array}\right)=1 \text { and }\left(\begin{array}{l}
\lambda \\
v
\end{array}\right)=\frac{\lambda(\lambda-1) \cdots(\lambda-v+1)}{v !}=\frac{(\lambda)_{v}}{v !}(v \in \mathbb{N}, \lambda \in \mathbb{C})
$$

(cf. [2-38]). 
The Bernoulli numbers of first kind $B_{n}$ are defined by:

$$
\frac{t}{e^{t}-1}=\sum_{n=0}^{\infty} B_{n} \frac{t^{n}}{n !}
$$

where $|t|<2 \pi(c f .[2-38])$.

The Euler numbers of first kind $E_{n}$ are defined by:

$$
\frac{2}{e^{t}+1}=\sum_{n=0}^{\infty} E_{n} \frac{t^{n}}{n !}
$$

where $|t|<\pi$ (cf. [2-38]).

The Euler polynomials of second kind $E_{n}^{*}(x)$ are defined by:

$$
\frac{2}{e^{t}+e^{-t}} e^{t x}=\sum_{n=0}^{\infty} E_{n}^{*}(x) \frac{t^{n}}{n !},
$$

where $|t|<\frac{\pi}{2}$. For $x=0$, we have:

$$
E_{n}^{*}=E_{n}^{*}(0),
$$

which denotes the Euler numbers of the second kind (cf. [2-38]).

Let $k \in \mathbb{N}_{0}$. The Stirling numbers of the second kind $S_{2}(n, k)$ are defined by:

$$
\frac{\left(e^{t}-1\right)^{k}}{k !}=\sum_{n=0}^{\infty} S_{2}(n, k) \frac{t^{n}}{n !},
$$

Using Equation (2), we have $S_{2}(n, k)=0$ if $n<k$ or $k<0$ (cf. [2-38]).

The Stirling numbers of the first kind $S_{1}(n, k)$ are defined by:

$$
\frac{(\log (1+t))^{k}}{k !}=\sum_{n=0}^{\infty} S_{1}(n, k) \frac{t^{n}}{n !}
$$

Using Equation (3), we have $S_{1}(n, k)=0$ if $k>n$ or $k<0$ (cf. [2-38]).

The $\lambda$-array polynomials $S_{k}^{n}(x ; \lambda)$ are defined by:

$$
\frac{1}{k !} e^{t x}\left(\lambda e^{t}-1\right)^{k}=\sum_{n=0}^{\infty} S_{k}^{n}(x ; \lambda) \frac{t^{n}}{n !}
$$

(cf. $[2,6,30])$.

The Bernoulli numbers of the second kind (or the Cauchy numbers of the first kind) $b_{n}(0)$ are defined by:

$$
\frac{t}{\log (1+t)}=\sum_{n=0}^{\infty} b_{n}(0) \frac{t^{n}}{n !}
$$

The numbers $b_{n}(0)$ are also given by:

$$
b_{n}(0)=\int_{0}^{1}(u)_{n} d u
$$

(cf. [26] (p. 116)).

The central factorial numbers of the second kind $T(n, k)$ are defined by:

$$
\frac{1}{(2 k) !}\left(e^{t}+e^{-t}-2\right)^{k}=\sum_{n=0}^{\infty} T(n, k) \frac{t^{2 n}}{(2 n) !}
$$


(cf. $[4,8,9])$.

Operators $\mathcal{O}_{\lambda}[f ; a, b]$ and $T_{\lambda}[f ; a, b]$

Let $a, x \in \mathbb{R}$ and

$$
E^{a}[f](x)=f(x+a)
$$

(cf. $[1,2,9,11,27,36,37])$. The operators $\mathcal{O}_{\lambda}[f ; a, b]$ and $T_{\lambda}[f ; a, b]$ are as follows, respectively:

$$
\mathcal{O}_{\lambda}[f ; a, b](x)=\lambda E^{a}[f](x)+E^{b}[f](x),
$$

and

$$
T_{\lambda}[f ; a, b](x)=\frac{\mathcal{O}_{\lambda}[f ; a, b](x)}{a+b+1}
$$

where $\lambda, a$, and $b$ are real parameters. (cf. [1]). In [1], the following special cases of the operator $T_{\lambda}[f ; a, b]$ were provided. These special cases have many different applications in mathematics, engineering, etc.:

$$
\begin{aligned}
\frac{1}{2} T_{1}[f ; 0,0](x) & =I[f](x), \text { (Identity Operator) } \\
-2 T_{-1}[f ; 1,0](x) & =\Delta[f](x), \text { (Forward Difference Operator) } \\
I[f](x)+\frac{1}{2} T_{1}[f ;-1,-1](x) & =\nabla[f](x), \text { (Backward Difference Operator) } \\
T_{1}[f ; 1,0](x) & =M[f](x), \text { (Means Operator) } \\
-T_{-1}\left[f ; \frac{1}{2},-\frac{1}{2}\right](x) & =\delta[f](x), \text { (Central Difference Operator) } \\
\frac{1}{2} T_{1}\left[f ; \frac{1}{2},-\frac{1}{2}\right](x) & =\mu[f](x),(\text { Averaging Difference Operator) } \\
-(2 a+b+1) T_{-1}[f ; a+b, a](x) & =\Delta_{b} E^{a}[f](x),(a \neq b, \text { Gould Operator) } \\
-2 T_{-\lambda}[f ; 1,0](x) & =\Delta_{\lambda}[f](x) .
\end{aligned}
$$

For details about the above operators and their applications, see [1,3-36].

The remainder of this paper is structured as follows: Section 2 outlines a new finite operator is defined. Some properties of this operator are given. In Section 3, using this new operator, two new classes of special polynomials and numbers are defined. The derivative formulas for these new polynomials are given. In Section 4, some integral representations related to the Volkenborn integral, the fermionic $p$-adic integral, and the Riemann integral for these new polynomials are given. Using these integral representations, many new identities and formulas are derived including the Bernoulli numbers, the Euler numbers, the Stirling numbers, the Daehee numbers, and the Changhee numbers. Finally, Section 5 provides the conclusions.

\section{A New Operator}

In this section, we define a new operator that modifies the operators $\mathcal{O}_{\lambda}[f ; a, b]$ and $T_{\lambda}[f ; a, b]$. Some properties of this operator are given.

Let $a$ and $b$ be real parameters. Let $\lambda$ and $\beta$ be real or complex parameters. A new operator $\mathbb{Y}_{\lambda, \beta}[f ; a, b]$ is defined by:

$$
\mathbb{Y}_{\lambda, \beta}[f ; a, b](x)=\lambda E^{a}[f](x)+\beta E^{b}[f](x) .
$$

Some special values of this operator are given as follows:

$$
\mathbb{Y}_{\lambda, \beta}[f ; a, b](x)=\beta \mathcal{O}_{\frac{\lambda}{\beta}}[f ; a, b](x)
$$


and

$$
\mathbb{Y}_{\lambda, \beta}[f ; a, b](x)=\beta(a+b+1) T_{\frac{\lambda}{\beta}}[f ; a, b](x)
$$

(cf. [1]).

$$
\mathbb{Y}_{-\lambda, 1}[f ; 1,0](x)=-\Delta_{\lambda} f(x)=-\lambda f(x+1)+f(x)
$$

(cf. [2]).

$$
\begin{gathered}
\mathbb{Y}_{1,0}[f ; a, 0](x)=E^{a}[f](x) . \\
\mathbb{Y}_{1,-1}[f ; a, 0](x)=\Delta_{a}[f](x)=f(x+a)-f(x),
\end{gathered}
$$

where $\Delta_{a}$ denotes the forward difference operator.

$$
\begin{aligned}
\mathbb{Y}_{1,-1}[f ; 0,-b](x) & =\nabla_{-b}[f](x) \\
& =\left(\mathbb{Y}_{1,-1}[f ; b, 0] \mathbb{Y}_{1,0}[f ;-b, 0]\right)[f](x) \\
& =\left(\mathbb{Y}_{1,-1}[f ; b, 0] \mathbb{Y}_{1,0}[f ; 0,-b]\right)[f](x) \\
& =\left(E^{b}-I\right) E^{-b}[f](x) \\
& =f(x)-f(x-b),
\end{aligned}
$$

where $\nabla_{-b}$ denotes the backward difference operator.

$$
\begin{aligned}
\mathbb{Y}_{1,-1}\left[f ; \frac{a}{2},-\frac{a}{2}\right](x) & =\delta_{a}[f](x) \\
& =\left(E^{\frac{a}{2}}-E^{-\frac{a}{2}}\right)[f](x) \\
& =f\left(x+\frac{a}{2}\right)-f\left(x-\frac{a}{2}\right),
\end{aligned}
$$

where $\delta_{a}$ denotes the central difference operator.

$$
\begin{aligned}
\mathbb{Y}_{1,-1}\left[f ; \frac{a}{2},-\frac{a}{2}\right] & =\left(\mathbb{Y}_{1,-1}[f ; a, 0]\right) \mathbb{Y}_{1,0}\left[f ;-\frac{a}{2}, 0\right] \\
& =\left(\mathbb{Y}_{1,-1}[f ; 0,-a]\right) \mathbb{Y}_{1,0}\left[f ; \frac{a}{2}, 0\right] \\
& =\delta_{a}[f]
\end{aligned}
$$

(cf. [2-36]). The Gould operator is:

$$
\mathbb{Y}_{1,0}[f ; a+b, 0]-\mathbb{Y}_{1,0}[f ; a, 0]=G_{a, b}[f],
$$

where $a \neq b$ (cf. [25]).

$$
{ }_{a} \Delta^{1}=\frac{\mathbb{Y}_{1,0}[f ; a, 0]-I[f]}{a}
$$

(cf. [11] (p. 27, Equation (1.17))).

The operator $\nabla[f]$ is provided by Goldstine [11] (p. 128). Applying this operator to $f(x)=E_{n}^{*}(x)$, we have:

$$
\nabla[f](x)=\frac{\mathbb{Y}_{1,0}[f ; 1,0](x)+f(x)}{2}=x^{n}
$$

where $n \in \mathbb{N}_{0}$. Therefore,

$$
\begin{aligned}
\sum_{k=0}^{m-1}(-1)^{k} k^{n} & =\frac{1}{2} \sum_{k=0}^{m-1}(-1)^{k}\left(\mathbb{Y}_{1,0}[f ; 1,0](k)+f(k)\right) \\
& =\frac{1}{2}\left(E_{n}^{*}(0)-(-1)^{m} E_{n}^{*}(m)\right),
\end{aligned}
$$


(cf. [11] (p. 136)).

Let,

$$
\mathbb{Y}_{\lambda, \beta}^{k}[f ; a, b]=\mathbb{Y}_{\lambda, \beta}[f ; a, b]\left(\mathbb{Y}_{\lambda, \beta}^{k-1}[f ; a, b]\right)
$$

where $k \in \mathbb{N}$.

By applying the operator in Equation (10) $k$-times to the function $f$, and using Equation (11), we obtain:

$$
\mathbb{Y}_{\lambda, \beta}^{k}[f ; a, b](x)=\sum_{j=0}^{k}\left(\begin{array}{c}
k \\
j
\end{array}\right) \lambda^{k-j} \beta^{j} f(x+j b+(k-j) a) .
$$

Remark 1. Substituting $b=0$ and $\beta=-1$ into (12), we have:

$$
\begin{aligned}
\mathbb{Y}_{\lambda,-1}^{k}[f ; 1,0](x) & =\sum_{j=0}^{k}\left(\begin{array}{l}
k \\
j
\end{array}\right) \lambda^{k-j}(-1)^{j} f(x+(k-j) a) \\
& =\Delta_{\lambda}^{k}[f](x)
\end{aligned}
$$

(cf. [2] (p. 155, Equation (29))).

Remark 2. Substituting $f(x)=x^{n}, b=0$, and $\beta=-1$ into Equation (12), the polynomials $\mathbb{Y}_{\lambda,-1}^{k}\left[x^{n} ; 1,0\right](0)$ reduce to $\lambda$-array polynomials $S_{k}^{n}(x ; \lambda)$ :

$$
\begin{aligned}
\mathbb{Y}_{\lambda,-1}^{k}\left[x^{n} ; 1,0\right](x) & =\Delta_{\lambda}^{k}\left[x^{n}\right](x) \\
& =S_{k}^{n}(x ; \lambda)
\end{aligned}
$$

(cf. [2] (p. 155)).

Substituting $a=\frac{1}{2}, b=-\frac{1}{2}, \lambda=1$ and $\beta=-1$ into Equation (12), we have:

$$
\delta[f](x)=\mathbb{Y}_{1,-1}^{k}\left[f ; \frac{1}{2},-\frac{1}{2}\right](x)=\sum_{j=0}^{k}(-1)^{j}\left(\begin{array}{l}
k \\
j
\end{array}\right) f\left(x-j+\frac{k}{2}\right) .
$$

Substituting $f(x)=x^{n}\left(n \in \mathbb{N}_{0}\right)$ into the previous equation, we have:

$$
\mathbb{Y}_{1,-1}^{k}\left[x^{n} ; \frac{1}{2},-\frac{1}{2}\right](x)=\sum_{j=0}^{k}(-1)^{j}\left(\begin{array}{l}
k \\
j
\end{array}\right)\left(x-j+\frac{k}{2}\right)^{n} .
$$

Let:

$$
\mathbb{Y}_{\lambda, \beta}^{j}[f ; a, b](0)=\left.\mathbb{Y}_{\lambda, \beta}^{j}[f ; a, b](x)\right|_{x=0} .
$$

Substituting $x=0$ into Equation (13), we obtain:

$$
T(n, k)=\frac{1}{k !} \mathbb{Y}_{1,-1}^{k}\left[x^{n} ; \frac{1}{2},-\frac{1}{2}\right](0) .
$$

where $n, k \in \mathbb{N}_{0}$ and:

$$
\delta[f](0)=\mathbb{Y}_{1,-1}^{k}\left[x^{n} ; \frac{1}{2},-\frac{1}{2}\right](0) .
$$

Equation (14) is also provided in ([4] Equation (2.8)), [20,32].

\section{New Families of Special Polynomials and Numbers}

In this section, we define two new classes of special polynomials and numbers. 
Substituting $f(x)=\sum_{l=1}^{n} d_{l} x^{l}\left(n \in \mathbb{N}_{0}\right.$ and $\left.d_{l} \in \mathbb{R}\right)$ into (12), we define a new class of special polynomials as follows:

$$
P_{n}(x, k ; a, b ; \lambda, \beta, d)=\sum_{l=1}^{n} d_{l} \mathbb{Y}_{\lambda, \beta}^{k}\left[x^{l} ; a, b\right](x),
$$

where,

$$
d=\left(d_{1}, d_{2}, \ldots, d_{n}\right)
$$

Therefore,

$$
P_{n}(x, k ; a, b ; \lambda, \beta, d)=\sum_{j=0}^{k}\left(\begin{array}{l}
k \\
j
\end{array}\right) \lambda^{k-j} \beta^{j} \sum_{l=1}^{n}(x+j b+(k-j) a)^{l} d_{l} .
$$

Observe that for $\beta=1$, Equation (15) is unification of Equation (21) in [1]. Using Equation (15), we obtain:

$$
P_{n}(x, k ; a, b ; \lambda, \beta, d)=\sum_{j=0}^{k}\left(\begin{array}{l}
k \\
j
\end{array}\right) \lambda^{k-j} \beta^{j} \sum_{l=1}^{n} d_{l} \sum_{v=0}^{l}\left(\begin{array}{l}
l \\
v
\end{array}\right) x^{v}(j b+(k-j) a)^{l-v}
$$

After some elementary calculations, we obtain:

$$
P_{n}(x, k ; a, b ; \lambda, \beta, d)=\sum_{l=1}^{n} \sum_{v=0}^{l}\left(\begin{array}{l}
l \\
v
\end{array}\right) d_{l} x^{v} \mathbb{Y}_{\lambda, \beta}^{k}\left[x^{l-v} ; a, b\right](0) .
$$

With the help of the previous equation, a new class of special numbers can now be defined as follows:

$$
y_{5}(n, k ; a, b ; \lambda, \beta)=\sum_{l=1}^{n} d_{l} \mathbb{Y}_{\lambda, \beta}^{k}\left[x^{l} ; a, b\right](0) .
$$

Combining the above definition with Equation (12), we have:

$$
y_{5}(n, k ; a, b ; \lambda, \beta)=\sum_{j=0}^{k}\left(\begin{array}{c}
k \\
j
\end{array}\right) \lambda^{k-j} \beta^{j} \sum_{l=1}^{n} d_{l}(j b+(k-j) a)^{l} .
$$

Observe that for $\beta=1$, Equation (16) is unification of Equation (22) in [1].

By applying the derivative operator $\frac{d^{k}}{d x^{k}}$ to Equation (15), we obtain the derivative formula for polynomials $P_{n}(x, k ; a, b ; \lambda, \beta)$ as follows:

$$
\frac{d^{k}}{d x^{k}}\left\{P_{n}(x, k ; a, b ; \lambda, \beta, d)\right\}=\sum_{j=0}^{k}\left(\begin{array}{c}
k \\
j
\end{array}\right) \lambda^{k-j} \beta^{j} \sum_{l=1}^{n}(l)_{k}(x+j b+(k-j) a)^{l-k} d_{l} .
$$

Combining the above equation with the following well-known formula (cf. [26]):

$$
(y)_{k}=\sum_{j=0}^{k} S_{1}(k, j) y^{j}
$$

we obtain a derivative formula for the polynomials $P_{n}(x, k ; a, b ; \lambda, \beta)$ by the following theorem:

Theorem 1. Let $n \in \mathbb{N}$ and $k \in \mathbb{N}_{0}$ with $k \leq n$. Then, we have:

$$
\frac{d^{k}}{d x^{k}}\left\{P_{n}(x, k ; a, b ; \lambda, \beta, d)\right\}=\sum_{j=0}^{k}\left(\begin{array}{c}
k \\
j
\end{array}\right) \lambda^{k-j} \beta^{j} \sum_{l=1}^{n} d_{l} \sum_{m=0}^{k} S_{1}(k, m) l^{m}(x+j b+(k-j) a)^{l-k} .
$$


We now define another class of special polynomials as follows:

$$
Q_{n}(x ; a, b ; \lambda, \beta)=\sum_{j=0}^{n}\left(\begin{array}{l}
x \\
j
\end{array}\right) \mathbb{Y}_{\lambda, \beta}^{j}[f ; a, b](0) .
$$

In Equation (19), we assume that the values $f(0)$ and $f(m b+(j-m) a)$ are finite.

Using Equation (19), we obtain:

$$
Q_{n}(x ; a, b ; \lambda, \beta)=\sum_{j=0}^{n} \sum_{m=0}^{j}\left(\begin{array}{c}
x \\
j
\end{array}\right)\left(\begin{array}{c}
j \\
m
\end{array}\right) \lambda^{j-m} \beta^{m} f(m b+(j-m) a) .
$$

Some special values of the polynomials $Q_{n}(x ; a, b ; \lambda, \beta)$ are given as follows:

Substituting $n=2, f(y)=e^{y}$, and

$$
\mathbb{Y}_{\lambda, \beta}^{j}\left[e^{y} ; a, b\right](0)=\sum_{m=0}^{j}\left(\begin{array}{c}
j \\
m
\end{array}\right) \lambda^{j-m} \beta^{m} e^{m b+(j-m) a}
$$

into Equation (19), we have:

$$
\begin{aligned}
Q_{2}(x ; a, b ; \lambda, \beta)= & 1+x \sum_{m=0}^{1}\left(\begin{array}{c}
1 \\
m
\end{array}\right) \lambda^{1-m} \beta^{m} e^{m b+(1-m) a} \\
& +x^{2} \sum_{m=0}^{2}\left(\begin{array}{c}
2 \\
m
\end{array}\right) \lambda^{2-m} \beta^{m} e^{m b+(2-m) a}
\end{aligned}
$$

Therefore,

$$
Q_{2}(x ; a, b ; \lambda, \beta)=1+\left(\lambda e^{a}+\beta e^{b}-\left(\lambda e^{a}+\beta e^{b}\right)^{2}\right) x+\left(\lambda e^{a}+\beta e^{b}\right)^{2} x^{2} .
$$

Substituting $\lambda=1, \beta=-1$, and $b=-a$ into (21), we have:

$$
\begin{aligned}
Q_{2}(x ; a,-a ; 1,-1) & =1+2 x(\sinh (a)-\cosh (2 a)+1)+4 x^{2} \sinh ^{2}(a) \\
& =1+2 x\left(\sinh (a)-2 \sinh ^{2}(a)\right)+4 x^{2} \sinh ^{2}(a) .
\end{aligned}
$$

Substituting $\lambda=1, \beta=-1$, and $b=a$ into (21), we have:

$$
Q_{2}(x ; a, a ; 1,-1)=1+2 x e^{2 a} .
$$

Substituting $a=0$ into the aforementioned equation, we have:

$$
Q_{2}(x ; 0,0 ; 1,-1)=1+2 x .
$$

Substituting $f(x)=x^{n}, a=\lambda=1$ and $\beta=-1$ and $b=0$ into Equation (20), we obtain:

$$
Q_{n}(x ; 1,0 ; 1,-1)=\sum_{j=0}^{n}\left(\begin{array}{l}
x \\
j
\end{array}\right) j ! S_{2}(n, j) .
$$

Therefore,

$$
Q_{n}(x ; 1,0 ; 1,-1)=x^{n} .
$$

Combining Equations (22) and (17), we obtain the following corollary: 
Corollary 1. Let $n \in \mathbb{N}_{0}$. Then, we have:

$$
Q_{n}(x ; 1,0 ; 1,-1)=\sum_{j=0}^{n} \sum_{l=0}^{j} S_{2}(n, j) S_{1}(j, l) x^{l} .
$$

Derivative Formula for Polynomials $Q_{n}(x ; a, b ; \lambda, \beta)$

Here, we provide a derivative formula for the polynomials $Q_{n}(x ; a, b ; \lambda, \beta)$.

Taking derivative of Equation (19) with respect to $x$, and using the following well-known derivative formula for the function $\left(\begin{array}{l}x \\ n\end{array}\right)(c f .[24,33])$ :

$$
\frac{d}{d x}\left\{\left(\begin{array}{l}
x \\
n
\end{array}\right)\right\}=\left(\begin{array}{l}
x \\
n
\end{array}\right) \sum_{m=0}^{n-1} \frac{1}{x-m}
$$

we obtain:

$$
\begin{aligned}
\frac{d}{d x}\left\{Q_{n}(x ; a, b ; \lambda, \beta)\right\} & =\frac{d}{d x}\left\{\sum_{j=0}^{n}\left(\begin{array}{c}
x \\
j
\end{array}\right) \mathbb{Y}_{\lambda, \beta}^{j}[f ; a, b](0)\right\} \\
& =\sum_{j=1}^{n} \frac{\mathbb{Y}_{\lambda, \beta}^{j}[f ; a, b](0)}{j !}(x)_{j} \sum_{m=0}^{j-1} \frac{1}{x-m} .
\end{aligned}
$$

After some elementary calculations in the above equation, we arrive at the following theorem:

Theorem 2. Let $n \in \mathbb{N}$. Then, we have:

$$
\frac{d}{d x}\left\{Q_{n}(x ; a, b ; \lambda, \beta)\right\}=\sum_{j=1}^{n}\left(\begin{array}{c}
x \\
j
\end{array}\right) \mathbb{Y}_{\lambda, \beta}^{j}[f ; a, b](0) \sum_{m=0}^{j-1} \frac{1}{x-m} .
$$

\section{Integral Representations for the Polynomials $Q_{n}(x ; a, b ; \lambda, \beta)$}

In this section, we provide the Riemann integral and $p$-adic integrals representations for the polynomials $Q_{n}(x ; a, b ; \lambda, \beta)$. Using these integrals representations, many new identities and formulas are derived including combinatorial sums, the Bernoulli numbers, the Euler numbers, the Stirling numbers, the Daehee numbers, and the Changhee numbers.

\subsection{Riemann Integral Formulas of Polynomials $Q_{n}(x ; a, b ; \lambda, \beta)$}

Here, we provide some integral formulas for $Q_{n}(x ; a, b ; \lambda, \beta)$ polynomials. Using these formulas, some new identities and combinatorial sums are derived including the Stirling numbers and the Bernoulli numbers of the second kind.

Integrating Equation (20) from 0 to 1 and using ( refLamdaFun-1p), we obtain:

$$
\int_{0}^{1} Q_{n}(x ; a, b ; \lambda, \beta) d x=\sum_{j=0}^{n} \sum_{m=0}^{j}\left(\begin{array}{c}
j \\
m
\end{array}\right) \frac{b_{j}(0) \lambda^{j-m} \beta^{m} f(m b+(j-m) a)}{j !} .
$$

Integrating Equations (22)-(24) from 0 to 1, we obtain:

$$
\int_{0}^{1} Q_{n}(x ; 1,0 ; 1,-1) d x=\sum_{j=0}^{n} S_{2}(n, j) b_{j}(0),
$$




$$
\int_{0}^{1} Q_{n}(x ; 1,0 ; 1,-1) d x=\frac{1}{n+1},
$$

and

$$
\int_{0}^{1} Q_{n}(x ; 1,0 ; 1,-1) d x=\sum_{j=0}^{n} \sum_{l=0}^{j} \frac{1}{l+1} S_{2}(n, j) S_{1}(j, l) .
$$

Combining Equations (26) and (28), we arrive at the following theorem:

Theorem 3. Let $n, k \in \mathbb{N}_{0}$. Then, we have:

$$
\sum_{j=0}^{n} \sum_{l=0}^{j} \frac{1}{l+1} S_{2}(n, j) S_{1}(j, l)=\sum_{j=0}^{n} S_{2}(n, j) b_{j}(0) .
$$

Remark 3. Considering the method reported by Simsek and Cakic [34], using the orthogonality relation of the Stirling numbers, Equation (29) reduces to the following well-known relation:

$$
\sum_{j=0}^{n} S_{2}(n, j) b_{j}(0)=\frac{1}{n+1}
$$

(cf. $[5,7,9,12,25,26,34-37])$. Additionally, by combining (26) with (27), we obtain Equation (30).

\section{2. $p$-Adic Integrals Formulas of the Polynomials $Q_{n}(x ; a, b ; \lambda, \beta)$}

Here, by applying $p$-adic integrals to the polynomials $Q_{n}(x ; a, b ; \lambda, \beta)$, many $p$-adic integral formulas are derived. Using these $p$-adic integral formulas, some new combinatorial sums including the Bernoulli numbers, the Euler numbers, the Stirling numbers, the Daehee numbers, and the Changhee numbers are given.

We need the following definitions and notations for $p$-adic integrals:

Let $\mathbb{Z}_{p}$ and $\mathbb{Q}_{p}$ denote the set of $p$-adic integers and the set of $p$-adic rational numbers, respectively. Let $\mathbb{C}_{p}$ denote the field of $p$-adic completion of algebraic closure of $\mathbb{Q}_{p}$. Let $f: \mathbb{Z}_{p} \rightarrow \mathbb{C}_{p}$ be a uniformly differentiable function. $C^{1}\left(\mathbb{Z}_{p} \rightarrow \mathbb{C}_{p}\right)$ denotes a set of uniformly differentiable functions.

Let $f \in C^{1}\left(\mathbb{Z}_{p} \rightarrow \mathbb{C}_{p}\right)$. The Volkenborn integral of the function $f$ on $\mathbb{Z}_{p}$ is defined by:

$$
\int_{\mathbb{Z}_{p}} f(x) d \mu_{1}(x)=\lim _{N \rightarrow \infty} \frac{1}{p^{N}} \sum_{x=0}^{p^{N}-1} f(x),
$$

where $\mu_{1}(x)$ denotes the Haar distribution:

$$
\mu_{1}(x)=\frac{1}{p^{N}}
$$

(cf. $[16,18,19,27,32,38])$.

The fermionic $p$-adic integral of the function $f$ is defined by:

$$
\int_{\mathbb{Z}_{p}} f(x) d \mu_{-1}(x)=\lim _{N \rightarrow \infty} \sum_{x=0}^{p^{N}-1}(-1)^{x} f(x),
$$

where:

$$
\mu_{-1}(x)=(-1)^{x}
$$

(cf. [17-19] see also [21,32]). 
Some $p$-adic integrals formulas are given as follows:

$$
B_{n}=\int_{\mathbb{Z}_{p}} x^{n} d \mu_{1}(x),
$$

(cf. $[16,17,27,32])$.

$$
\int_{\mathbb{Z}_{p}}\left(\begin{array}{l}
x \\
n
\end{array}\right) d \mu_{1}(x)=\frac{(-1)^{n}}{n+1}=\frac{D_{n}}{n !},
$$

where $D_{n}$ denotes the Daehee numbers (cf. $\left.[14,27,32]\right)$.

$$
E_{n}=\int_{\mathbb{Z}_{p}} x^{n} d \mu_{-1}(x)
$$

(cf. [17]).

$$
\int_{\mathbb{Z}_{p}}\left(\begin{array}{l}
x \\
n
\end{array}\right) d \mu_{-1}(x)=(-1)^{n} 2^{-n}=\frac{C h_{n}}{n !},
$$

where $C h_{n}$ denotes the Changhee numbers ( $c f$. ([15] Theorem 2.3), [32]).

By applying the Volkenborn integral to Equation (20), using Equations (33) and (34), we obtain the following results, respectively:

$$
\begin{gathered}
\int_{\mathbb{Z}_{p}} Q_{n}(x ; a, b ; \lambda, \beta) d \mu_{1}(x)=\sum_{j=0}^{n} \sum_{m=0}^{j}\left(\begin{array}{c}
j \\
m
\end{array}\right) \frac{(-1)^{j}}{j+1} \lambda^{j-m} \beta^{m} f(m b+(j-m) a), \\
\int_{\mathbb{Z}_{p}} Q_{n}(x ; a, b ; \lambda, \beta) d \mu_{1}(x)=\sum_{j=0}^{n} \sum_{m=0}^{j}\left(\begin{array}{c}
j \\
m
\end{array}\right) \frac{D_{j}}{j !} \lambda^{j-m} \beta^{m} f(m b+(j-m) a),
\end{gathered}
$$

and

$$
\int_{\mathbb{Z}_{p}} Q_{n}(x ; a, b ; \lambda, \beta) d \mu_{1}(x)=\sum_{j=0}^{n} \sum_{m=0}^{j} \frac{1}{j !} \sum_{l=0}^{j}\left(\begin{array}{c}
j \\
m
\end{array}\right) S_{1}(j, l) B_{l} \lambda^{j-m} \beta^{m} f(m b+(j-m) a) .
$$

Combining Equations (37) and (39), we obtain the following theorem:

Theorem 4. Let $n \in \mathbb{N}_{0}$. Then, we have:

$$
\begin{aligned}
& \sum_{j=0}^{n} \sum_{m=0}^{j}\left(\begin{array}{c}
j \\
m
\end{array}\right) \frac{(-1)^{j}}{j+1} \lambda^{j-m} \beta^{m} f(m b+(j-m) a) \\
= & \sum_{j=0}^{n} \sum_{m=0}^{j} \frac{1}{j !} \sum_{l=0}^{j}\left(\begin{array}{c}
j \\
m
\end{array}\right) S_{1}(j, l) B_{l} \lambda^{j-m} \beta^{m} f(m b+(j-m) a) .
\end{aligned}
$$

Combining Equations (38) and (39), we obtain the following theorem:

Theorem 5. Let $n \in \mathbb{N}_{0}$. Then we have

$$
\begin{aligned}
& \sum_{j=0}^{n} \sum_{m=0}^{j}\left(\begin{array}{c}
j \\
m
\end{array}\right) \frac{D_{j}}{j !} \lambda^{j-m} \beta^{m} f(m b+(j-m) a) \\
= & \sum_{j=0}^{n} \sum_{m=0}^{j} \frac{1}{j !} \sum_{l=0}^{j}\left(\begin{array}{c}
j \\
m
\end{array}\right) S_{1}(j, l) B_{l} \lambda^{j-m} \beta^{m} f(m b+(j-m) a) .
\end{aligned}
$$


Substituting $a=1, b=0, \lambda=1, \beta=-1$ into (40), we arrive at the following result:

Corollary 2. Let $n \in \mathbb{N}_{0}$. Then, we have:

$$
\sum_{j=0}^{n} \sum_{l=0}^{j} B_{l} S_{2}(n, j) S_{1}(j, l)=\sum_{j=0}^{n} S_{2}(n, j) D_{j}
$$

Remark 4. Using the orthogonality relation of the Stirling numbers, Equation (41) reduces to the following well-known formula:

$$
B_{n}=\sum_{j=0}^{n} S_{2}(n, j) D_{j}
$$

$(c f .[14,26,32,34])$.

By applying the fermionic $p$-adic to Equation (20), using Equations (35) and (36), we obtain the following results, respectively:

$$
\begin{gathered}
\int_{\mathbb{Z}_{p}} Q_{n}(x ; a, b ; \lambda, \beta) d \mu_{-1}(x)=\sum_{j=0}^{n} \sum_{m=0}^{j}\left(\begin{array}{c}
j \\
m
\end{array}\right) \frac{(-1)^{j}}{2^{j}} \lambda^{j-m} \beta^{m} f(m b+(j-m) a), \\
\int_{\mathbb{Z}_{p}} Q_{n}(x ; a, b ; \lambda, \beta) d \mu_{-1}(x)=\sum_{j=0}^{n} \sum_{m=0}^{j}\left(\begin{array}{c}
j \\
m
\end{array}\right) \frac{\lambda^{j-m} \beta^{m} f(m b+(j-m) a) C h_{j}}{j !},
\end{gathered}
$$

and

$$
\int_{\mathbb{Z}_{p}} Q_{n}(x ; a, b ; \lambda, \beta) d \mu_{-1}(x)=\sum_{j=0}^{n} \sum_{m=0}^{j} \frac{1}{j !} \sum_{l=0}^{j}\left(\begin{array}{c}
j \\
m
\end{array}\right) S_{1}(j, l) E_{l} \lambda^{j-m} \beta^{m} f(m b+(j-m) a) .
$$

Combining Equations (42) and (44), we arrive at the following theorem:

Theorem 6. Let $n \in \mathbb{N}_{0}$. Then, we have:

$$
\begin{aligned}
& \sum_{j=0}^{n} \sum_{m=0}^{j}\left(\begin{array}{c}
j \\
m
\end{array}\right) \frac{(-1)^{j}}{2^{j}} \lambda^{j-m} \beta^{m} f(m b+(j-m) a) \\
= & \sum_{j=0}^{n} \sum_{m=0}^{j} \frac{1}{j !} \sum_{l=0}^{j}\left(\begin{array}{c}
j \\
m
\end{array}\right) S_{1}(j, l) E_{l} \lambda^{j-m} \beta^{m} f(m b+(j-m) a) .
\end{aligned}
$$

Combining Equations (42) and (44), we obtain the following theorem:

Theorem 7. Let $n \in \mathbb{N}_{0}$. Then, we have:

$$
\begin{aligned}
& \sum_{j=0}^{n} \sum_{m=0}^{j}\left(\begin{array}{c}
j \\
m
\end{array}\right) \frac{\lambda^{j-m} \beta^{m} f(m b+(j-m) a) C h_{j}}{j !} \\
= & \sum_{j=0}^{n} \sum_{m=0}^{j} \frac{1}{j !} \sum_{l=0}^{j}\left(\begin{array}{c}
j \\
m
\end{array}\right) S_{1}(j, l) E_{l} \lambda^{j-m} \beta^{m} f(m b+(j-m) a) .
\end{aligned}
$$

Substituting $a=1, b=0, \lambda=1, \beta=-1$ into Equation (45), we obtain the following corollary: 
Corollary 3. Let $n \in \mathbb{N}_{0}$. Then, we have:

$$
\sum_{j=0}^{n} \sum_{l=0}^{j} E_{l} S_{2}(n, j) S_{1}(j, l)=\sum_{j=0}^{n} S_{2}(n, j) C h_{j} .
$$

Remark 5. Using the orthogonality relation of the Stirling numbers, Equation (46) reduces to the following well-known formula:

$$
E_{n}=\sum_{j=0}^{n} S_{2}(n, j) C h_{j}
$$

(cf. $[12,15,26,32,34])$.

\section{Conclusions}

This paper introduced a new operator and new two classes of special polynomials and numbers. Many properties of this new operator, polynomials, and numbers were outlined. Using this operator, some special values of these special numbers and polynomials were derived. Many fundamental properties of these numbers and polynomials were investigated. $p$-adic integrals and the Riemann integral representations for these polynomials were provided. Using these integral representations, identities and formulas were derived including combinatorial sums, the Bernoulli numbers, the Euler numbers, the Stirling numbers, the Daehee numbers, and the Changhee numbers. The results of this paper may potentially be used in mathematics, physics, and engineering.

Acknowledgments: The present investigation was supported by Scientific Research Project Administration of Akdeniz University. The author would like to thank the referees for their suggestions and comments.

Funding: This research received no external funding.

Conflicts of Interest: The author declares no conflict of interest.

\section{References}

1. Simsek, Y. Construction method for generating functions of special numbers and polynomials arising from analysis of new operators. Math. Meth. Appl. Sci. 2018, 41, 6934-6954. [CrossRef]

2. Bayad, A.; Simsek, Y.; Srivastava, H.M. Some array type polynomials associated with special numbers and polynomials. Appl. Math. Comput. 2014, 244, 149-157. [CrossRef]

3. Boyadzhiev, K.N. Binomial transform and the backward difference. arXiv 2014, arXiv:1410.3014v2.

4. Butzer, P.L.; Schmidt, K.; Stark, E.L.; Vogt, L. Central factorial numbers; their main properties and some applications. Numer. Funct. Anal. Optim. 1989, 10, 419-488. [CrossRef]

5. Cakic, N.P.; Milovanovic, G.V. On generalized Stirling numbers and polynomials. Math. Balk. 2004, 18, 241-248.

6. Chang, C.-H.; Ha, C.-W. A multiplication theorem for the Lerch zeta function and explicit representations of the Bernoulli and Euler polynomials. J. Math. Anal. Appl. 2006, 315, 758-767. [CrossRef]

7. Charalambides, C.A. Ennumerative Combinatorics; Chapman\&Hall/CRC, Press Company: London, UK; New York, NY, USA, 2002.

8. Cigler, J. Fibonacci polynomials and central factorial numbers. Preprint. Available online: https://homepage. univie.ac.at/johann.cigler/preprints/central-factorial.pdf (accessed on 20 December 2019).

9. Comtet, L. Advanced Combinatorics: The Art of Finite and Infinite Expansions; Nienhuys Reidel, J.W., Translated; Springer Science \& Business Media: Berlin, Germany, 1974.

10. Djordjevic, G.B.; Milovanovic, G.V. Special Classes of Polynomials; University of Nis, Faculty of Technology Leskovac: Niš, Serbia, 2014.

11. Goldstine, H.H. A History of Numerical Analysis from the 16th through the 19th Century; Springer: New York, NY, USA; Heidelberg/Berlin, Germany, 1977.

12. Jordan C.; Carver, H. C. Calculus of Finite Differences, 2nd ed.; Chelsea Publishing Company: New York, NY, USA, 1950. 
13. Kang J.; Ryoo, C. A research on the new polynomials involved with the central factorial numbers, Stirling numbers and others polynomials. J. Ineq. Appl. 2014, 26, 1-10. [CrossRef]

14. Kim, D.S.; Kim, T. Daehee numbers and polynomials. Appl. Math. Sci. (Ruse) 2013, 7, 5969-5976. [CrossRef]

15. Kim, D.S.; Kim, T.; Seo, J. A note on Changhee numbers and polynomials. Adv. Stud. Theor. Phys. 2013, 7, 993-1003. [CrossRef]

16. Kim, T. q-Volkenborn integration. Russ. J. Math. Phys. 2002, 19, 288-299.

17. Kim, T. $q$-Euler numbers and polynomials associated with $p$-adic $q$-integral and basic $q$-zeta function. Trend Math. Inf. Cent. Math. Sci. 2006, 9, 7-12.

18. Kim, T. $q$-Euler numbers and polynomials associated with $p$-adic $q$-integrals. J. Nonlinear Math. Phys. 2007, 14, 15-27. [CrossRef]

19. Kim, T. On the analogs of Euler numbers and polynomials associated with $p$-adic $q$-integral on $\mathbb{Z}_{p}$ at $q=-1$. J. Math. Anal. Appl. 2007, 331, 779-792. [CrossRef]

20. Kim, T.; Kim, D. S. Degenerate central factorial numbers of the second kind. Rev. R. Acad. Cienc. Exactas Fís. Nat. Ser. A Mat. RACSAM 2019, 113, 3359-3367. [CrossRef]

21. Kim, M.S. On Euler numbers, polynomials and related p-adic integrals. J. Number Theory 2009, 129, $2166-2179$. [CrossRef]

22. Knuth, D.E. The Art of Computer Programming, 3rd ed.; Fundamental Algorithms; Addison-Wesley: Boston, MA, USA, 1997; Volume 1, ISBN 0-201-89683-4.

23. Lupas, A. A conjecture related to the approximation operators of binomial type. Gen. Math. 1998, 6, 39-49.

24. Poon, S.S. Higher Derivatives of the Falling Factorial and Related Generalizations of the Stirling and Harmonic Numbers. arXiv 2014, arXiv:1401.2737.

25. Quaintance, J.; Gould, H.W. Combinatorial Identities for Stirling Numbers: The Unpublished Notes of H. W. Gould; World Scientific Publishing Co. Pte. Ltd.: Singapore, 2016.

26. Roman, S. The Umbral Calculus; Academic Press: New York, NY, USA, 1984.

27. Schikhof, W.H. Ultrametric Calculus: An Introduction to p-Adic Analysis; Cambridge Studies in Advanced Mathematics 4; Cambridge University Press: Cambridge, UK, 1984.

28. Simsek, Y. Special functions related to Dedekind-type DC-sums and their applications. Russ. J. Math. Phys. 2010, 17, 495-508. [CrossRef]

29. Simsek, Y. Identities associated with generalized Stirling type numbers and Eulerian type polynomials. Math. Comput. Appl. 2013, 18, 251-263. [CrossRef]

30. Simsek, Y. Generating functions for generalized Stirling type numbers, array type polynomials, Eulerian type polynomials and their alications. Fixed Point Theory Appl. 2013, 87, 343-1355.

31. Simsek, Y. New families of special numbers for computing negative order Euler numbers and related numbers and polynomials. Appl. Anal. Discrete Math. 2018, 12, 1-35. [CrossRef]

32. Simsek, Y. Explicit formulas for p-adic integrals: Approach to p-adic distributions and some families of special numbers and polynomials. Montes Taurus J. Pure Appl. Math. 2019, 1, 1-76.

33. Simsek, Y. Peters type polynomials and numbers and their generating functions: Approach with $p$-adic integral method. Math. Meth. Appl. Sci. 2019, 42, 7030-7046. [CrossRef]

34. Simsek, Y.; Cakic, N. Identities associated with Milne-Thomson type polynomials and special numbers. J. Inequal. Appl. 2018, 84, doi:10.1186/s13660-018-1679-x. [CrossRef] [PubMed]

35. Spiegel, M.R. Calculus of Finite Differences and Difference Equations; Schaum's Outline Series in Mathematics; McGraw-Hill Book Company: London, UK; Toronto, ON, Canada, 1971.

36. Spivey, M.Z. Combinatorial sums and finite differences. Discrete Math. 2007, 307, 3130-3146. [CrossRef]

37. Srivastava, H.M.; Choi, J. Zeta and q-Zeta Functions and Associated Series and Integrals; Elsevier Science Publishers: Amsterdam, The Netherlands; London, UK; New York, NY, USA, 2012.

38. Volkenborn, A. On Generalized p-adic Integration. Mém. Soc. Math. Fr. 1974, 39-40, 375-384. [CrossRef]

(C) 2020 by the authors. Licensee MDPI, Basel, Switzerland. This article is an open access article distributed under the terms and conditions of the Creative Commons Attribution (CC BY) license (http:/ / creativecommons.org/licenses/by/4.0/). 